\title{
Review: medical therapy with calcium channel blockers or $\alpha$ blockers helps patients to pass urinary stones
}

Hollingsworth JM, Rogers MA, Kaufman SR, et al. Medical therapy to facilitate urinary stone passage: a meta-analysis. Lancet 2006;368:1171-9.

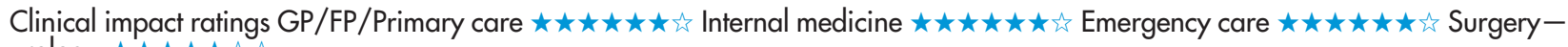

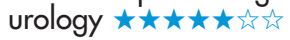

In patients with urolithiasis, are calcium channel blockers or $\alpha$ blockers more effective than standard therapy for helping patients pass urinary stones?

\section{METHODS}

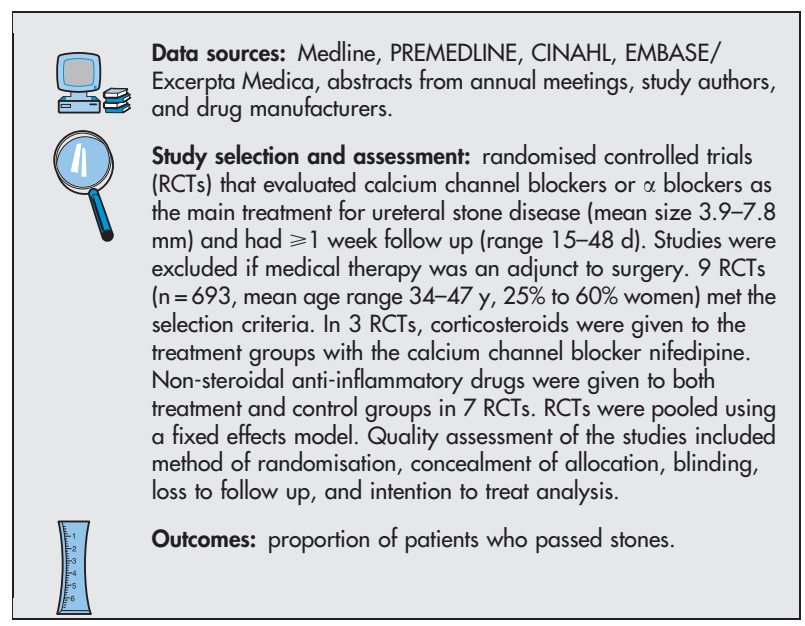

\section{MAIN RESULTS}

More patients in the treatment group than in the control group passed stones (table). 5 additional RCTs did not have a true control comparator (ie, calcium channel blockers or $\alpha$ blockers were compared with a treatment that was potentially active [eg, corticosteroids]); when these RCTs were added to the meta-analysis, the effect for medical therapy with calcium channel blockers or $\alpha$ blockers remained (relative benefit increase 52\%, 95\% CI 39 to 65 ). Mean time to passage ranged from 6 days in several treatment groups to 20 days in 1 control group.
For correspondence: Dr B K Hollenbeck, Ann Arbor Veterans Affairs Health Services Research and Development Center of Excellence, Ann Arbor, MI, USA. bhollen@umich.edu

Sources of funding: National Institutes of Health and Blue Cross Blue Shield of Michigan Foundation

\section{CONCLUSION}

In patients with urolithiasis, medical therapy with calcium channel blockers or $\alpha$ blockers may increase the chance of passing ureteral stones.

\section{Commentary}

U reteral colic is an extremely painful and costly medical condition. Despite this, surprisingly few RCTs have examined non-invasive methods to speed stone passage. Traditionally, increased fluids (orally or intravenously) and pain control have been the mainstay of treatment. However, the benefits of increased fluids are uncertain. ${ }^{1}$ While urological intervention is nearly always effective, the associated discomfort, risks, and costs preclude this treatment as routine first line therapy.

The meta-analysis by Hollingsworth et al highlights 2 inexpensive and widely available medications that increased the likelihood of stone passage. However, several issues should be considered. Firstly, in the setting of uncontrollable pain or infection, emergent urological intervention should not be delayed. Secondly, the long term effect on renal function of prolonged partial or complete ureteral obstruction needs clarification. It is not only the proportion of stones that eventually pass but also the time to passage that matters. Thirdly, the role of the other therapies used with the study drugs is uncertain. For several studies, $\geqslant 1$ intervention was used, thus making it difficult to separate the independent effects of the drugs of interest. Fourthly, the costs associated with delaying the urological intervention need to be determined. For example, do patients require more analgesics and still have to miss work while waiting for the stone to pass? Fiffhly, while the title of the meta-analysis refers to "medical therapy," it only addresses the role of calcium channel blockers and $\alpha$ blockers. In fact, the only calcium channel blocker studied was nifedipine and the results cannot necessarily be extrapolated to other calcium channel blockers. Finally, the authors conclude that "a highquality randomized trial is necessary to confirm the efficacy of [these drugs]," implying that the studies included in the meta-analysis were not "high-quality." I agree that this research question warrants further study because if these drugs are truly effective, they should become part of the standard treatment of ureteral stone disease. Based on the available evidence, it seems reasonable to try nifedipine or an $\alpha$ blocker to facilitate ureteral stone passage while waiting for definitive studies.

Gary C Curhan, MD, ScD

Brigham and Women's Hospital Boston, Massachusetts, USA

1 Worster A, Richards C. Fluids and diuretics for acute ureteric colic. Cochrane Database Syst Rev 2005;(3):CD004926.

Medical therapy (calcium channel blockers or $\alpha$ blockers) $v$ no medical therapy for urolithiasis at 15-48 days*

\begin{tabular}{lllllll}
\hline & & \multicolumn{3}{c}{ Weighted event rates } & & \\
\cline { 5 - 6 } Outcome & Number of trials $(\mathbf{n})$ & Medical therapy & No medical therapy & Weighted RBI (95\% Cl) & NNT (CI) \\
\hline Urinary stone passage & $9(693)$ & $78 \% \dagger$ & $47 \%$ & $65 \%(45$ to 88$)$ & $4(3$ to 4$)$ \\
\hline
\end{tabular}

*Abbreviations defined in glossary.

tWeighted event rate and CI for NNT calculated from data in article. A fixed effects model was used. 